\title{
Water Management using Remotely Operated Vehicle for Aquaculture
}

\author{
Udhayachandrika $\mathbf{N}^{1}$, Koushika Vema ${ }^{2}$, Shalini Kannan ${ }^{3}$, Shanthakumari $G^{4}$ \\ ${ }^{1}$ UG - Electronics and Communication Engineering, Sri Sairam Engineering College, Tamil Nadu \\ ${ }^{2}$ UG - Electronics and Communication Engineering, Sri Sairam Engineering College, Tamil Nadu \\ ${ }^{3}$ UG - Electronics and Communication Engineering, Sri Sairam Engineering College, Tamil Nadu \\ ${ }^{4}$ Assistant Professor, Electronics and Communication Engineering, Sri Sairam Engineering College, Tamil Nadu
}

\begin{abstract}
In the aquaculture industry, fish, shrimp, and aquatic plants are cultivated in fresh, salt, or brackish waters. The increasing demand for aquatic products has stimulated the increasing growth of aquaculture industries. The physical parameters of water quality concerns that affect aquaculture cultivation are $\mathrm{pH}$, temperature, and turbidity. The formation of ammonia, hydrogen sulphide, algae blooms, and excretion of waste by species have high chances to affect the proportion of physical parameters in water and there is a risk of life threat to the species present there. A surface boat enabled with a wireless sensor network and embedded technology is designed. By navigating the surface boat, regular monitoring of these physical parameters can be displayed and accessed through a mobile application. The proposed system can help aquaculture organizations operate in a real-time environment for optimum monitoring and control of the aquaculture environment.
\end{abstract}

Keywords: Water Quality, Aquaculture, Microcontroller.

\section{INTRODUCTION}

Overfishing of oceans and other natural resources is continuously increasing over the year, humans need alternate sources for seafood to feed the planet's growing population. Aquaculture is the process of cultivating aquatic plants or animals, especially for human consumption. Aquaculture is also known as fish farming. Fish farming is a responsible and sustainable solution to provide a solution for future generations with access to healthy and environmentally friendly protein options. Regardless of the particular aquaculture system used, maintaining balanced levels of water quality parameters like temperature, $\mathrm{pH}$ and turbidity are fundamental for both the health and growth of farmed aquatic species. In our system, real-time monitoring of the physical parameters of water and displaying through a mobile application is done to maintain the aquaculture environment friendly.

\section{EXPERIMENTAL METHODS OR METHODOLOGY}

\subsection{System Design}

A surface boat enabled with a wireless sensor network and embedded technology is designed. By navigating the surface boat through the entire water body, the real-time sensors attached to the surface boat collect real-time values of $\mathrm{pH}$, temperature, and turbidity of the water. The collected real-time values are transmitted wirelessly to the mobile application using IoT technology. The proposed system can help aquaculture organizations operate in a real-time environment for optimum monitoring and control of the aquaculture environment.

\subsection{Vehicle Control Setup}

The Arduino UNO is the microcontroller of this setup, which controls the functions of the vehicle. The Arduino UNO is powered using Lithium-ion Battery, whose voltage is controlled by the voltage regulator. The operations performed in Flysky-i6 Transmitter will be reflected in the Flysky-i6 Receiver. The Arduino UNO acts as an interface between ESC (Electronic Speed Controller) and Receiver and manages their functions. The Electronic Speed Controller controls and regulates the speed of the Thruster. Thus, the vehicle moves. 


\section{International Advanced Research Journal in Science, Engineering and Technology}

Vol. 8, Issue 6, June 2021

DOI: $10.17148 / I A R J S E T .2021 .8621$

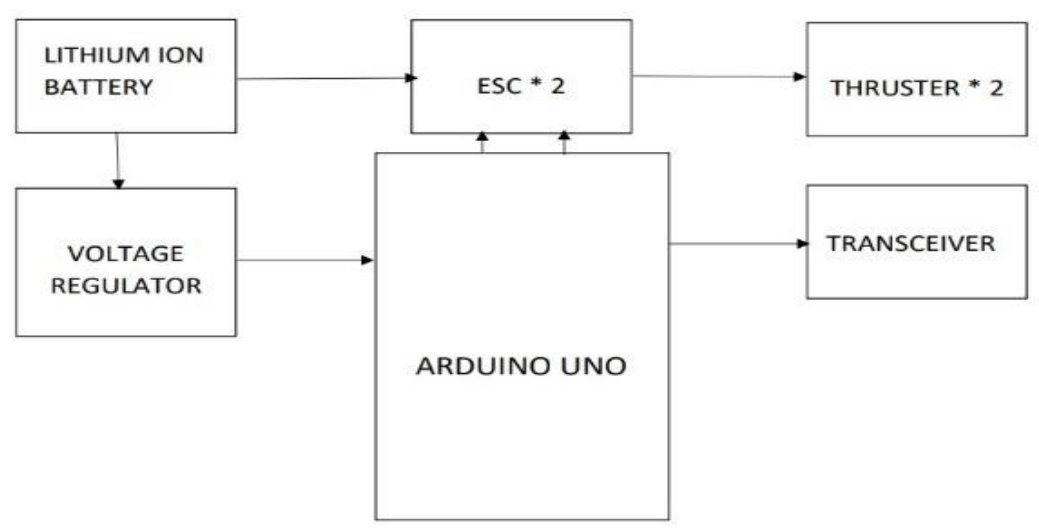

Fig. 1. Block diagram of Vehicle Control Setup

\subsection{Sensor Setup}

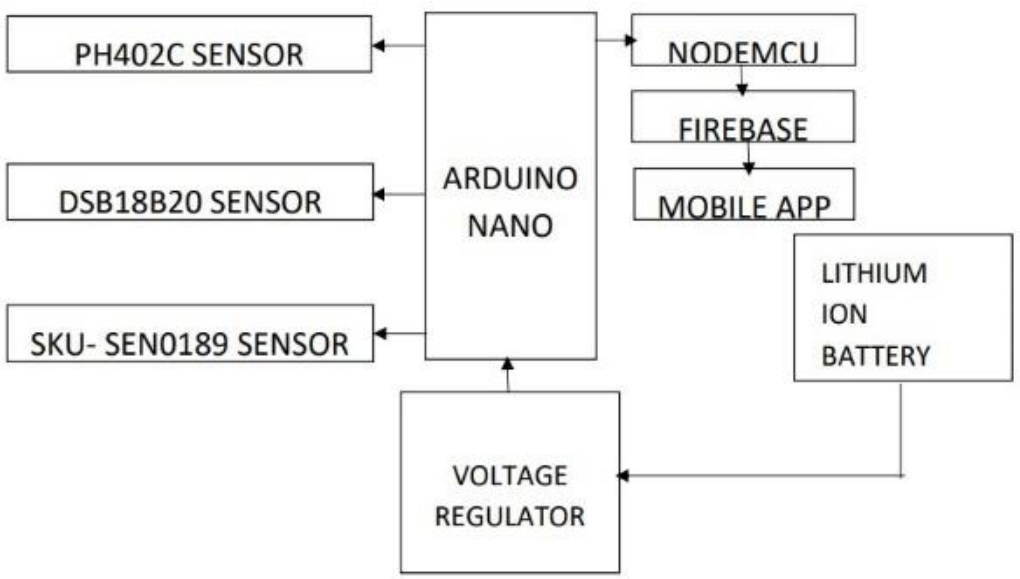

Fig. 2. Block diagram of Sensor Setup

The PH402C sensor (pH Sensor), DSB18B20 sensor (Temperature Sensor), and SKU-SEN0189 sensor (Turbidity Sensor) are connected to the Arduino Nano. The Arduino Nano is the microcontroller of this setup, which controls the functions of the sensors and the NodeMCU. The Arduino Nano is powered using Lithium-ion Battery, whose voltage is controlled by the voltage regulator. The Firebase acts as an interface and a communicator between NodeMCU and Mobile App. Through these means, the sensor data is received by the Mobile App.

\subsection{Working Principle}

IoT-enabled Smart Pond Monitoring System has been designed and developed to periodically monitor the quality of water to reduce high risks associated with aquaculture farming. Our real-time monitoring system gives a dramatic rise to Aquaculture farming and productivity.

The Surface boat is deployed on the pond at the starting location. The remote-controlled 2.4ghz transmitter allows the surface boat to cover a range of about $1.8 \mathrm{kms}$. After the deployment, the surface boat is maneuvered around the pond. The T200 thruster attached to the surface boat is connected with the Electronic Speed Controller (ESC). The electronic speed controller is a MOSFET attached to the system which controls the T200 Thruster. The sensor hub present in the surface boat collects the real-time values and sends the data to the IoT center. The collected sensor values will be displayed on the mobile application. The Arduino Uno is open-source hardware based on the Microchip ATmega328P microcontroller. It is developed by Arduino. cc. The Arduino UNO microcontroller board consists of digital and analog I/O pins that may be interfaced to other boards and circuits. The ESC and the receiver are attached with the Arduino UNO.

The transmitter is triggered to arm the surface vehicle. After arming the transmitter, it sends the signals to the receiver. The receiver receives the command and sends it to the Arduino UNO. The Arduino UNO processes the signal and sends 


\section{International Advanced Research Journal in Science, Engineering and Technology}

Vol. 8, Issue 6, June 2021

DOI: 10.17148/IARJSET.2021.8621

it to the Electronic Speed Controller to control the T200 Thruster. Thus, the surface boat is maneuvered with the signals corresponding to the transmitter.

While maneuvering, the water quality of the pond ecosystem is measured using the sensor hub in the surface boat. The sensor hub consists of a Ds18b20 sensor, $\mathrm{pH}$ sensor, and turbidity sensor, and these sensors are connected to Arduino nano. The Arduino Nano is open-source hardware based on the Microchip ATmega328P microcontroller. It is developed by Arduino. cc. The Arduino nano microcontroller board consists of digital and analog I/O pins that may be interfaced to other boards and circuits. Ds18b20 measures temperature of the water, pH sensor measures the acidity level present in the water, turbidity sensor measures the amount of light that is scattered by the suspended solids in water which is all connected to Arduino nano to process all the sensors and store the values.

After retrieving the data from the sensor hub, the real-time measured values are sent directly to the IoT center using NodeMCU. NodeMCU is an open-source firmware and development kit that initially runs on the ESP8266 Wi-Fi SoC and hardware based on the ESP-12 module and support for the ESP32 32-bit MCU was added.

The NodeMCU sends all the real-time values received from the sensor hub to the IoT server Firebase. Firebase is a Backend-as-a-Service (Baas). It provides developers with a variety of tools and services to develop quality apps, grows their user base, and earn profit. Firebase is a NoSQL database program, which stores data in the form of JSON-like documents. Firebase from Google is used to create web and mobile applications. Firebase sends all the data to our designed app retrieving data from it.

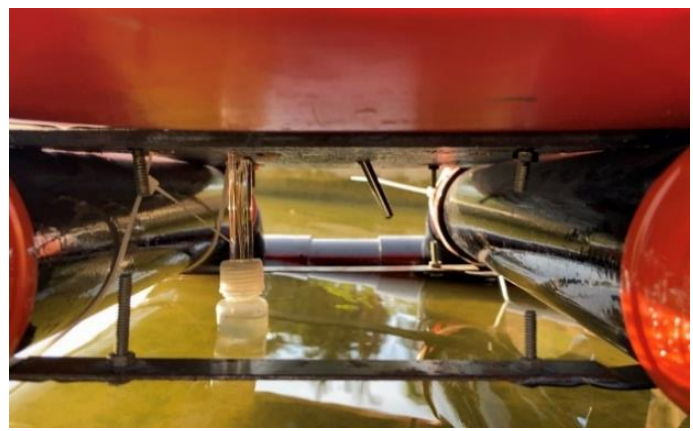

Fig. 3. Sensor Unit

\section{RESULTS AND DISCUSSION}

\subsection{Observation}

Table.4. Optimum values of Fish and Shrimp Farming Vs Values Obtained through Mobile Application

\begin{tabular}{|c|c|c|}
\hline Name & Range for Shrimp and Fish Farming & Obtained Results \\
\hline \multirow{2}{*}{ pH Sensor } & Shrimp: 6-8.5 & \\
\hline Temperature Sensor & Fish: $5.5-9.0$ & $6.7-7.9$ \\
\hline & $\begin{array}{c}\text { Shrimp: } 78.8-86 \mathrm{~F} \\
\text { Fish: 75-90F }\end{array}$ & $87.5-95 \mathrm{~F}$ \\
\hline Turbidity Sensor & Clean: $100-200 \mathrm{FTU}$ & $700-850 \mathrm{FTU}$ \\
& Cloudy:500-600 FTU & \\
\hline
\end{tabular}

\subsection{Results Obtained from the Mobile Application}

The sensor data acquired by the boat is received through the developed mobile application at a regular interval of 12 seconds. After logging in, the following will be displayed. 
International Advanced Research Journal in Science, Engineering and Technology

Vol. 8, Issue 6, June 2021

DOI: 10.17148/IARJSET.2021.8621

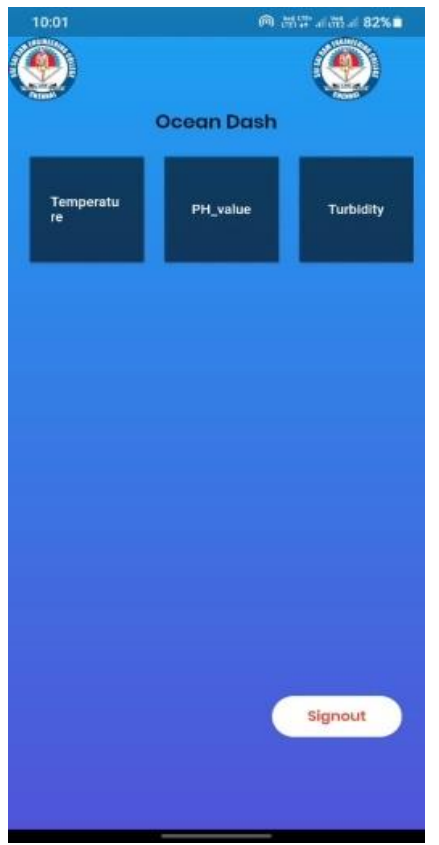

Fig. 5. Water Quality Parameter Icons

By clicking any water quality parameter icon, its corresponding values at regular intervals (12 seconds) will be displayed as shown below.

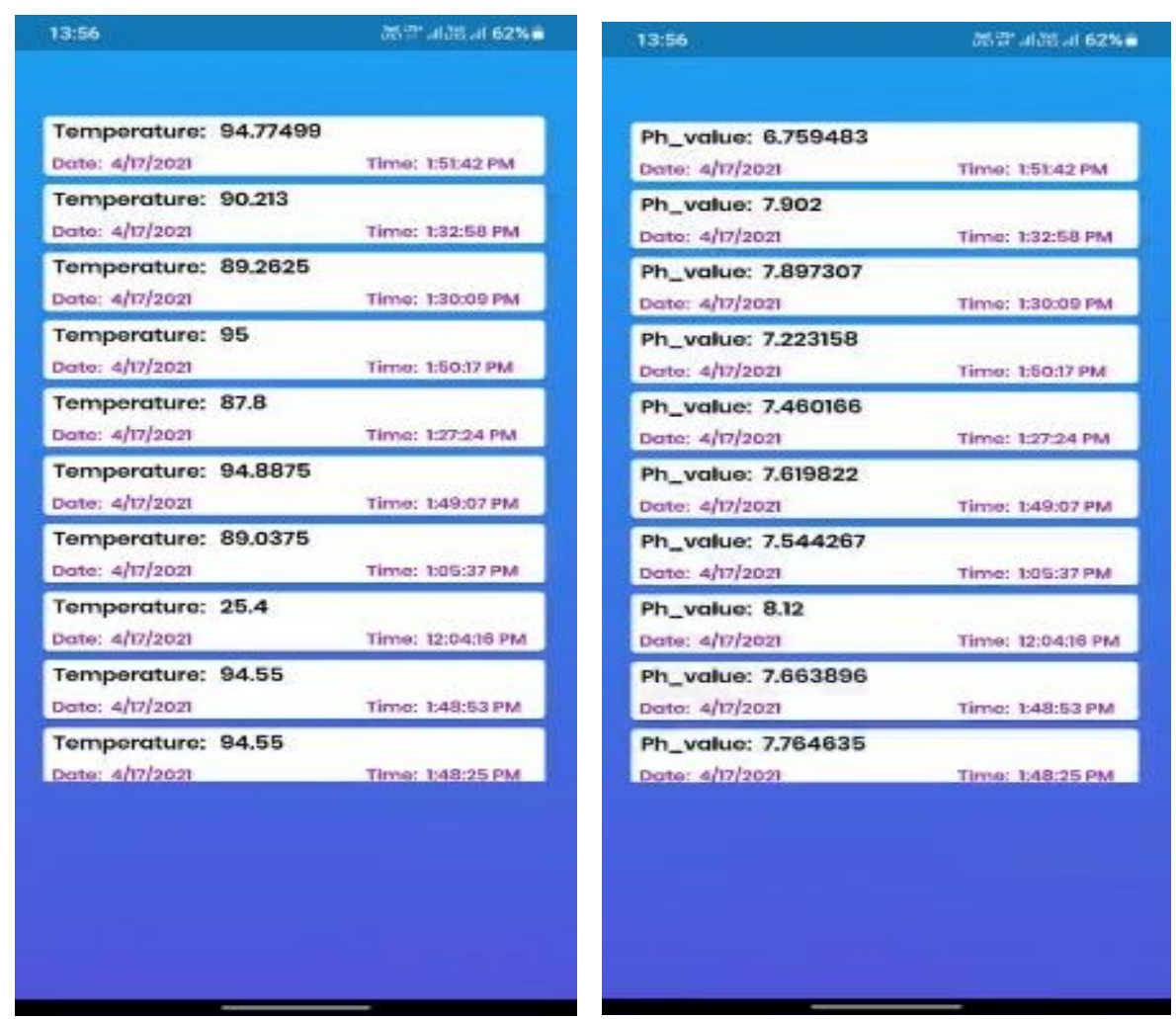

Fig. 6. Results Obtained (Temperature and pH Values) 


\section{International Advanced Research Journal in Science, Engineering and Technology}

Vol. 8, Issue 6, June 2021

DOI: 10.17148/IARJSET.2021.8621

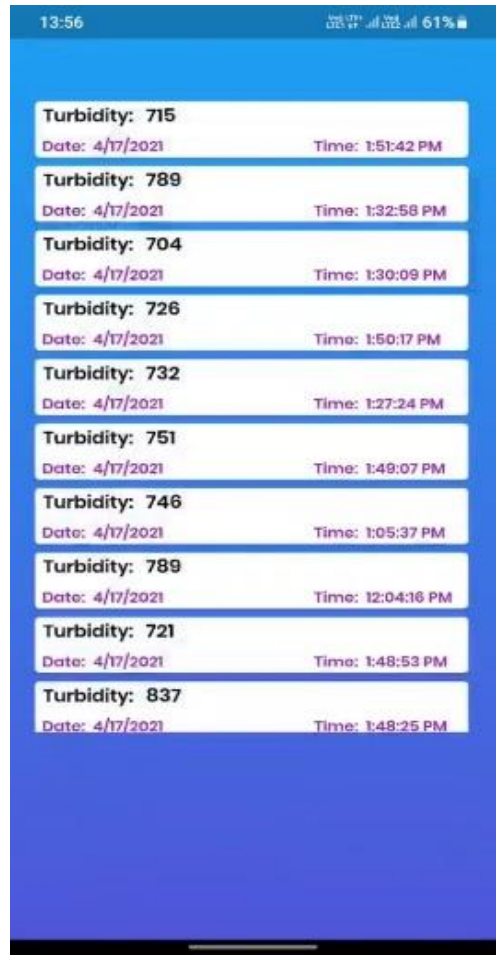

Fig. 7. Results Obtained (Turbidity Values)

\section{CONCLUSION}

In this system, we have developed a surface boat enabled with a wireless sensor network and embedded technology. The surface boat designed with sensors helps monitor the chemical and physical properties of water like $\mathrm{pH}$, temperature, and turbidity, and the collected data is displayed through the mobile application. This system can help aquaculture organizations operate in a real-time environment for optimum monitoring and control of the aquaculture environment, protection of the environment, improvement in water quality, reduction in water pollution.

\section{FUTURE SCOPE}

- The proposed work can be further designed and programmed to convert the remotely operated vehicle to an autonomous vehicle. Thus, no manual work will be required.

- This surface boat can be integrated with some more sensors to monitor other water quality parameters like salinity, dissolved oxygen, electrical conductivity, moisture etc.

- Interfacing GPS module can monitor the exact location of pollution and necessary action in that particular water location can be taken.

\section{REFERENCES}

[1]. Chien, Yew-Hu; "Water Quality Requirement and Management for Marine Shrimp Culture”. Proceedings of the Special Session on Shrimp Farming. 1992, page 144 .

[2]. Daudi S. Simbeye and Shi Feng Yang, Water Quality Monitoring and Control for Aquaculture Based on Wireless Sensor Networks. Journal of Networks, 2014, Vol.9, No.4

[3]. Huang Jianqing, Wang Weixing, Jiang Sheng, et al. Development and test of aquacultural water quality monitoring system based on wireless sensor network[J]. Transactions of the Chinese Society of Agricultural Engineering, 2013, 29(4): 183-190.

[4]. LI Caigen. Pond water quality criterion and control[J]. Scientific planting,2007(6).

[5]. Li Chengchun. Design of Wireless Multi-parameter Sensor Testing Network Based on CC2430[D]. Zhenjiang: Jiangsu University,2010.

[6]. M. Zennaro, A. Floros, G. Dogan, T. Sun, Z. Cao, C. Huang, et al., "On the design of a Water Quality Wireless Sensor Network (WQWSN): an Application to Water Quality Monitoring in Malawi", pp. 330-336, 22-25 Sept. 2009.

[7]. S. Mijovic and B. Palmar, "Water Quality Monitoring Automation of Rivers in Serbia", Work. Liv. Env. Pro., vol. 9, no. 1, pp. 1-10, 2012.

[8]. A. Faustine and A. N. Mvuma, "Ubiquitous Mobile Sensing for Water Quality Monitoring and Reporting within Lake Victoria Basin", Wire. Sens. Net., vol. 6, no. 2014, pp. 257-264.

[9]. S. C. Mukhopadhyay and A. Mason, Smart Sensors for Real-Time Water Quality Monitoring, Springer, 2013.

[10]. X. Zhu, D. Li, D. He, J. Wang, D. Ma, and F. Li, "A remote wireless system for water quality online monitoring in intensive fish culture," 75 Computers and Electronics in Agriculture, vol. 71, no. 1, pp. S3-S9, Apr. 2010.

[11]. LuoHongpin, Li Guanglin, PengWeifeng, Song Jie and BaiQiuwei, Realtime Remote Monitoring System for Aquaculture Water Quality. Int J Agric\&BiolEng, 2015, Vol.8, No.6 